\title{
Accelerated Degradation Tests Modeling Based on the Nonlinear Wiener Process with Random Effects
}

\author{
Shengjin Tang, Xiaosong Guo, Chuanqiang Yu, Haijian Xue, and Zhijie Zhou \\ High-Tech Institute of Xian, Xian, Shaanxi 710025, China \\ Correspondence should be addressed to Chuanqiang Yu; yuchuanqiang201@126.com
}

Received 27 February 2014; Accepted 6 May 2014; Published 26 May 2014

Academic Editor: Erik Cuevas

Copyright (C) 2014 Shengjin Tang et al. This is an open access article distributed under the Creative Commons Attribution License, which permits unrestricted use, distribution, and reproduction in any medium, provided the original work is properly cited.

Accelerated degradation tests (ADT) modeling is an important issue in lifetime assessment to the products with high reliability and long lifetime. Among the literature about the accelerated nonlinear degradation process modeling, the current methods did not consider the product-to-product variation of the products with the same type. Therefore, this paper proposes an accelerated degradation process modeling method with random effects for the nonlinear Wiener process. Firstly, we derive the lifetime distribution of the nonlinear Wiener process with random effects. Secondly, the nonlinear Wiener process is used to model the degradation process of a single stress, and the drift coefficient is considered as a random variable to describe the product-to-product variation. Using the random acceleration model, the random effects are incorporated into the constant stress ADT models and the step stress ADT models. Then, a two-step maximum likelihood estimation (MLE) method is presented to estimate the unknown parameters in the degradation models. Finally, a simulation study and a case study are provided to demonstrate the application and superiority of the proposed model.

\section{Introduction}

In the accelerated tests, the products are tested at high levels of accelerating variables (e.g., current, temperature, voltage, or pressure) to obtain the estimates of life or longterm performance at normal conditions through a physically reasonable statistical model [1]. Thus, the accelerated tests are widely used in manufacturing industries and maintenances, for example, to assess the lifetime distribution of the products, derive the prior information for the real time remaining useful life estimation, apply in the burn-in test to eradicate manufacturing defects, [1-4], and so forth. Accelerated life tests (ALTs) are the traditional method to assess the lifetime distribution of the products. However, conducting an ALT is time-consuming and requires many time-to-failure data. Moreover, it is difficult to obtain enough time-to-failure data in a short period of testing time, especially for the highly reliable and long-lifetime products.

To overcome these difficulties, an alternative method called accelerated degradation tests (ADT) is to collect the quality characteristic data, whose degradation is highly correlated with the lifetime of the products. Compared with the ALT, ADT could provide more reliability information and be conducted more quickly $[1,5]$. The research focus on the ADT includes two main aspects, that is, degradation modeling and degradation tests optimization. As the ADT modeling is the basic of the degradation tests optimization, it has gained much attention in recent years [6]. The commonly used models for ADT modeling based on the stochastic processes are the Gamma process and Wiener process [7]. The Gamma process only adapts to the degradation process which is always positive and strictly increasing. However, many degradation processes are not strictly increasing.

The Wiener process has provided a feasible way to handle this problem [8]. The typical properties of the Wiener process are the nonmonotonic property, infinite divisibility property, and physical interpretations, which could provide a good description of system's dynamic characteristic [9]. Based on these typical properties, the Wiener process has been widely used to model the degradation processes, such as rotating element bearings [2], LEDs [4, 10-12], superluminescent diodes (SLD) [13], laser generators [14], bridge beams [15], gyros [16], capacitors [17], hard disk drives [18], and lithiumion batteries [19]. For the ADT modeling, Liao and Tseng [10] 
used the nonlinear Wiener process to model the step stress ADT (SSADT). Tseng and Peng [11] used the nonlinear Wiener process to model the constant stress ADT (CSADT). Similar works can be found in $[12,13]$. However, in these studies, the random effects are not incorporated into the ADT modeling.

The random effects have already been incorporated in the degradation path of a single stress. Peng and Tseng [14] incorporated the random effects into the modeling of the Wiener process by regarding the drift parameter of the Wiener process as a random value. Similarly, Wang [15] assumed that the drift parameter and the diffusion parameter are all random for the nonlinear Wiener process to represent the product-to-product variation among different products. Moreover, the random effects have already been incorporated into the ADT modeling with the Gamma process [20]; thus, it is necessary to incorporate the random effects into the ADT modeling with the Wiener process. Tang et al. [21] presented a model with random effects for the SSADT via the linear Wiener degradation process. However, the random effects have not been incorporated in the nonlinear accelerated degradation process, which exists widely in the degradation paths $[16,17]$.

For the degradation process with random effects, a classic work about estimating the unknown modeling parameters by the MLE method is presented by [14], which has further been applied to the nonlinear Wiener process [16] and the Wiener process with measurement error $[18,22,23]$. However, the drift parameter is assumed to be random for a specific item, which is not consistent with the modeling assumptions and could lead to negative estimation of the variance of the drift parameter. To solve this problem, Tang et al. [19] presented a two-step MLE method to ensure the consistence of the modeling assumptions. However, this method is only for the degradation data with a single stress.

From the above review of the related works, we observe that modeling the ADT for the nonlinear degradation process has not been studied thoroughly. There are two main issues remaining to be solved for modeling the nonlinear ADT. The first is to describe the relation between the stress and the degradation rate with random effects. The second is to estimate the unknown parameters in the model. To solve the first issue, we generalize the modeling method from the single stress to the ADT. As the method presented by Wang [15] needs the expectation maximization algorithm to estimate the model parameters, it is difficult to be generalized to the ADT. Thus, we only regard the drift parameter as the random value to represent the random effects, similar to $[14,16,21$, $23,24]$. Then, we present a nonlinear degradation model with random effects for the CSADT and SSADT. To solve the second issue, we propose a two-step maximum likelihood estimation (MLE) method to estimate the unknown parameters of the ADT data. Finally, the application and superiority of the proposed model are validated through a simulation study and a case study.

The remainder of this paper is organized as follows. Section 2 develops the degradation modeling of the nonlinear Wiener process and derives the lifetime distribution.
In Section 3, we incorporate the random effects into the CSADT and the SSADT. In Section 4, we propose a new MLE method to estimate the unknown parameters. A simulation study and a case study are provided in Section 5. Section 6 draws the main conclusions.

\section{Degradation Modeling and Lifetime Distribution Estimation}

2.1. Degradation Model. There are four main types of nonlinear Wiener process for the degradation modeling, that is, logtransformation $[2,25]$, time-scale transformation $[15,18,26]$, direct modeling $[16,23]$, and general Wiener process $[17,24]$. The time-transformed Wiener process is most commonly used to model the accelerated degradation data [10, 11, 27]. Therefore, in this paper, we use the time-transformed Wiener process to model the ADT data with random effects. The modeling of other types of Wiener process for the ADT data is similar. Let $X(t)$ denote the degradation value at time $t$; then the degradation process can be represented as follows:

$$
X(t)=\lambda \Lambda(t)+\sigma_{B} B(\Lambda(t)),
$$

where $\lambda$ is the drift parameter, $\Lambda(t)$ is a positive nondecreasing function (e.g., $\Lambda(t)=t^{\theta}$ and $\Lambda(t)=e^{\theta t}-1$ ), $\sigma_{B}$ is the diffusion parameter, and $B(t)$ is the standard Brownian motion which represents the stochastic dynamics of the degradation process. If $\Lambda(t)=t$, the nonlinear model becomes the traditional linear drift-based Wiener process which appeared in [14]. That is,

$$
X(t)=\lambda t+\sigma_{B} B(t) .
$$

In most degradation applications, there exists substantial product-to-product variability among the degradation processes of different individuals. Such heterogeneity in the degradation paths can be represented by the random effects of the degradation processes. As mentioned above, it is difficult to model the accelerated degradation processes if the $\lambda$ and $\sigma_{B}$ are both considered as the random parameters to represent the heterogeneity among different products. Therefore, in this paper, we only regard the drift parameter as the random parameter to represent the heterogeneity among different products. For simplicity, it is assumed that $\lambda$ follows the normal distribution, that is, $N\left(\mu_{\lambda}, \sigma_{\lambda}^{2}\right)$. In the following, we propose the lifetime distribution of the degradation model with random effects.

2.2. Derivation of the Lifetime Distribution. Generally, if $X(t)$ reaches a prespecified failure threshold $w$ for the first time, a product is announced to be failed. Then, under the concept of the first hitting time (FHT) of the degradation process, the lifetime of the Wiener process can be represented as

$$
T=\inf \{t: X(t) \geq w \mid X(0) \leq w\} .
$$

The main results of the lifetime distribution are summarized in the following proposition. 
Proposition 1. For the nonlinear degradation process as shown in (1), the probability density function (PDF) and the cumulative distribution function (CDF) of the lifetime can be obtained as follows:

$$
\begin{aligned}
f_{T}(t)= & \frac{w}{\sqrt{2 \pi \Lambda(t)^{3}\left(\sigma_{\lambda}^{2} \Lambda(t)+\sigma_{B}^{2}\right)}} \\
& \times \exp \left(-\frac{\left(w-\mu_{\lambda} \Lambda(t)\right)^{2}}{2 \Lambda(t)\left(\sigma_{\lambda}^{2} \Lambda(t)+\sigma_{B}^{2}\right)}\right) \frac{d \Lambda(t)}{d t}, \\
F_{T}(t)= & \Phi\left(\frac{\mu_{\lambda} \Lambda(t)-w}{\sqrt{\sigma_{B}^{2} \Lambda(t)+\sigma_{\lambda}^{2} \Lambda(t)^{2}}}\right) \\
& +\exp \left(\frac{2 \mu_{\lambda} w}{\sigma_{B}^{2}}+\frac{2 \sigma_{\lambda}^{2} w^{2}}{\sigma_{B}^{4}}\right) \\
& \times \Phi\left(\begin{array}{c}
\left.-\frac{2 \sigma_{\lambda}^{2} w \Lambda(t)+\sigma_{B}^{2}\left(w+\mu_{\lambda} \Lambda(t)\right)}{\sigma_{B}^{2} \sqrt{\sigma_{B}^{2} \Lambda(t)+\sigma_{\lambda}^{2} \Lambda(t)^{2}}}\right) .
\end{array} .\right.
\end{aligned}
$$

Proof. In the sense of the FHT, it is well known that the Wiener process crossing a constant threshold $w$ obeys an inverse Gaussian distribution [28]. Accordingly, the CDF of the lifetime for the linear model given in (2) can be obtained as follows:

$$
F_{T}(t)=\Phi\left(\frac{\lambda t-w}{\sqrt{\sigma_{B}^{2} t}}\right)+\exp \left(\frac{2 \lambda w}{\sigma_{B}^{2}}\right) \Phi\left(-\frac{(w+\lambda t)}{\sqrt{\sigma_{B}^{2} t}}\right)
$$

By considering the random effects, that is, $\lambda \sim N\left(\mu_{\lambda}, \sigma_{\lambda}^{2}\right)$, the CDF becomes [14]

$$
\begin{aligned}
F_{T}(t)= & \Phi\left(\frac{\mu_{\lambda} t-w}{\sqrt{\sigma_{B}^{2} t+\sigma_{\lambda}^{2} t^{2}}}\right) \\
& +\exp \left(\frac{2 \mu_{\lambda} w}{\sigma_{B}^{2}}+\frac{2 \sigma_{\lambda}^{2} w^{2}}{\sigma_{B}^{4}}\right) \\
& \times \Phi\left(-\frac{2 \sigma_{\lambda}^{2} w t+\sigma_{B}^{2}\left(w+\mu_{\lambda} t\right)}{\sigma_{B}^{2} \sqrt{\sigma_{B}^{2} t+\sigma_{\lambda}^{2} t^{2}}}\right)
\end{aligned}
$$

By setting $t=\Lambda(t)$ in the above equation, we obtain the $\mathrm{CDF}$ of the nonlinear Wiener process as shown in (5). Then, taking the first derivative of the CDF with $t$ gives the PDF in (4) after some algebraic operation. This completes the proof.

Note that if we set $\Lambda(t)=t$ in (4) and (5), the lifetime distribution of the nonlinear Wiener process can be reduced to the distribution of the linear model. This is as expected because the linear model is a special case of the nonlinear model. This result is desired since the analytical explicit form of the lifetime distribution is obtained. To gain the degradation information under the typical-use stress, the ADT should be modeled first. We address this issue in the following section.

\section{Accelerated Degradation Modeling}

In this section, we incorporate the random effects into the accelerated degradation test modeling. As the constant stress ADT (CSADT) and the step stress ADT (SSADT) are the two typical ways for implementing the ADT, these two types of ADT modeling with random effects are the main focus in this paper. In order to model the degradation path under ADT, the random acceleration model is first introduced in the following.

3.1. Random Acceleration Model. Acceleration model is used to describe the relation between the stress and the degradation rate [29]. The conventional acceleration model includes the power rule model, Arrhenius model, and Eyring model. The power rule model is usually applied for the dielectric breakdown of capacitors and fatigue testing of materials. And the Arrhenius model is commonly used for the thermals and semiconductor materials. For more details of the acceleration model, see [29]. To represent the product-to-product variability among different products, Tang et al. [21] proposed a random Arrhenius model for the linear SSADT. In this paper, we generalize the random Arrhenius model to the nonlinear case.

For the nonlinear degradation process given in (1), the conventional Arrhenius model can be expressed as

$$
\lambda(S)=a e^{-b / S},
$$

where $a$ and $b$ denote the constants and $S$ denotes the stress. It can be observed that the drift parameter is constant. To represent the product-to-product variability among different products, the drift is assumed as a random parameter as discussed previously. Then, we have

$$
\lambda(S)=\mathbf{a} e^{-b / S}, \quad \mathbf{a} \sim N\left(\mu_{a}, \sigma_{a}^{2}\right) .
$$

Note that if we assume that $\sigma_{a}^{2}=0$, then the random Arrhenius model turns to the conventional Arrhenius model. This is as expected since any properly developed random model should cover the constant model as its special case. Similarly, we can also incorporate the randomness into the power rule model and Eyring model, respectively, as follows:

$$
\begin{array}{cc}
\lambda(S)=\mathbf{a} S^{b}, & \mathbf{a} \sim N\left(\mu_{a}, \sigma_{a}^{2}\right), \\
\lambda(S)=\mathbf{a} S e^{-b / S}, & \mathbf{a} \sim N\left(\mu_{a}, \sigma_{a}^{2}\right) .
\end{array}
$$

3.2. Model the CSADT with Random Effects. For the CSADT, the products are divided into some groups and each group is tested in an accelerated stress. Compared with the SSADT, the CSADT needs more products to conduct an ADT efficiently. 
However, it gains more degradation information in one stress and is easy to extend to the case with more types of stress [11, 12]. Thus, it has been applied widely in practice, for example, LEDs [11, 12], OLEDs [30], and so forth. In the following, we incorporate the random effects into the CSADT and use the random Arrhenius model for an illustrative purpose.

Let $S_{1}, S_{2}, \ldots, S_{m}$ denote $m$ higher stress levels such that $S_{1}<S_{2}<\cdots<S_{m}$. Based on the random Arrhenius model, the drift parameter under stress $S_{i}$ can be represented as

$$
\lambda_{i}=\lambda\left(S_{i}\right)=\mathbf{a} e^{-b / S_{i}}, \quad \mathbf{a} \sim N\left(\mu_{a}, \sigma_{a}^{2}\right)
$$

It follows that

$$
\lambda_{i} \sim N\left(\mu_{a} e^{-b / S_{i}}, \sigma_{a}^{2} e^{-2 b / S_{i}}\right)
$$

Let $X\left(t \mid S_{i}\right)$ denote the degradation value at time $t$ under stress $S_{i}$; then the degradation process can be formulated as

$$
X\left(t \mid S_{i}\right)=\lambda_{i} \Lambda(t)+\sigma_{B} B(\Lambda(t)), \quad i=1,2, \ldots, m .
$$

3.3. Model the SSADT with Random Effects. For the SSADT, all the products are tested in the same accelerated stress, and the stress increases or decreases step by step from a level to a predefined level until an appropriate termination time is reached $[13,31,32]$. Obviously, the advantage of the SSADT is that only a few test units are needed for conducting an ADT. Thus, it is more efficient than the CSADT and has been applied to LEDs [10], SLDs [13], and so forth. The SSADT with random effects can be expressed as follows.

Suppose that the testing stress of an SSADT experiment can be expressed as follows, where $t_{i-1}$ and $t_{i}$ denote the initiative and terminal time under stress $S_{i}$, respectively:

$$
S= \begin{cases}S_{1} & 0 \leq t<t_{1} \\ S_{2} & t_{1} \leq t<t_{2} \\ \vdots & \\ S_{m} & t_{m-1} \leq t<t_{m} .\end{cases}
$$

For an illustrative purpose, we apply the random power rule model to represent the product-to-product variability among different products. Then, the drift parameter under stress $S_{i}$ can be represented as

$$
\lambda_{i}=\lambda\left(S_{i}\right)=\mathbf{a} S_{i}^{b}, \quad \mathbf{a} \sim N\left(\mu_{a}, \sigma_{a}^{2}\right) .
$$

Similar to the CSADT, the drift parameter also follows the normal distribution; that is,

$$
\lambda_{i} \sim N\left(\mu_{a} S_{i}^{b}, \sigma_{a}^{2} S_{i}^{2 b}\right)
$$

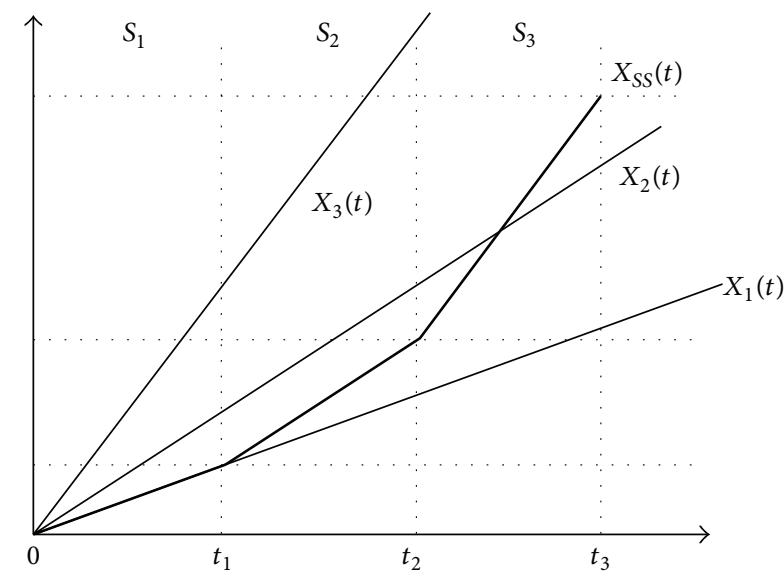

FIGURE 1: A typical transformed SSADT path with three stress levels.

Figure 1 shows a typical transformed SSADT plot with three stress levels. It can be observed that the starting degradation value under a new stress equals the termination value under the previous stress. Therefore, the degradation process of the SSADT can be expressed as follows:

$$
\begin{aligned}
& X_{S S}(t) \\
& =\left\{\begin{array}{rr}
\lambda_{1} \Lambda(t)+\sigma_{B} B(\Lambda(t)) & \\
0 \leq t<t_{1} & \\
\lambda_{2}\left(\Lambda(t)-\Lambda\left(t_{1}\right)\right)+\lambda_{1} t_{1}+\sigma_{B} B(\Lambda(t)) & t_{1} \leq t<t_{2} \\
\vdots & \\
\sum_{i=1}^{m-1} \lambda_{i}\left(\Lambda\left(t_{i}\right)-\Lambda\left(t_{i-1}\right)\right) & \\
& \lambda_{m}\left(\Lambda(t)-\Lambda\left(t_{m-1}\right)\right)+\sigma_{B} B(\Lambda(t)) \\
& t_{m-1} \leq t<t_{m} .
\end{array}\right.
\end{aligned}
$$

In the following, we develop a parameters estimation method to estimate the parameters in the proposed models.

\section{Parameters Estimation}

In this section, we use the two-step MLE method proposed in [19] to estimate the unknown parameters in the two ADT models. Without loss of generality, we set $\Lambda(t)=t^{\theta}$. The parameters estimation of other types of $\Lambda(t)$ is similar. As the random effects are considered in the ADT model, the unknown parameters are $\Phi=\left\{\mu_{a}, \sigma_{a}^{2}, b, \sigma_{B}^{2}, \theta\right\}$.

4.1. CSADT. It is assumed that there are $N$ tested products in all, and $n_{i}$ tested products under stress $S_{i}$. Thus, $N=\sum_{i=1}^{m} n_{i}$. At the experiment, each product is tested at time $t_{1}, t_{2}, \ldots, t_{L}$. 
Let $z_{i j k}$ denote the increment value for the $j$ th product at time $t_{k}$ under stress $S_{i}$; then we have

$$
\begin{aligned}
z_{i j k}= & X_{j}\left(t_{k} \mid S_{i}\right)-X_{j}\left(t_{k-1} \mid S_{i}\right) \\
= & \lambda_{i}\left(\Lambda\left(t_{k}\right)-\Lambda\left(t_{k-1}\right)\right)+\sigma_{B} B\left(\Lambda\left(t_{k}\right)-\Lambda\left(t_{k-1}\right)\right) \\
= & a_{j} e^{-b / S_{i}}\left(\Lambda\left(t_{k}\right)-\Lambda\left(t_{k-1}\right)\right)+\sigma_{B} B\left(\Lambda\left(t_{k}\right)-\Lambda\left(t_{k-1}\right)\right) \\
i=1,2, \ldots, m ; \quad k=1,2, \ldots, L ; & \quad \sum_{i=1}^{i-1} n_{i}<j \leq \sum_{i=1}^{i} n_{i},
\end{aligned}
$$

where $a_{j}$ denotes the specific value of the random parameter a for the $j$ th product.

Based on the independent increment property of the Wiener process, the $z_{i j k}$ obeys the normal distribution as follows:

$$
\begin{array}{r}
z_{i j k} \sim N\left(a_{j} e^{-b / S_{i}}\left(\Lambda\left(t_{k}\right)-\Lambda\left(t_{k-1}\right)\right),\right. \\
\left.\sigma_{B}^{2}\left(\Lambda\left(t_{k}\right)-\Lambda\left(t_{k-1}\right)\right)\right) .
\end{array}
$$

Let $\Theta=\left\{a_{1}, a_{2}, \ldots, a_{N}, b, \sigma_{B}^{2}, \theta\right\}$; then the log-likelihood function can be expressed as

$$
\begin{aligned}
\ln L( & \Theta \mid \mathbf{Z}) \\
= & -\frac{m N L}{2}\left(\ln (2 \pi)+\ln \left(\sigma_{B}^{2}\right)\right)-\frac{m N}{2} \\
& \times \sum_{k=1}^{L} \ln \left(\Lambda\left(t_{k}\right)-\Lambda\left(t_{k-1}\right)\right)-\frac{1}{2 \sigma_{B}^{2}} \\
& \times \sum_{i=1}^{m} \sum_{j=n_{i-1}+1}^{n_{i}} \sum_{k=1}^{L} \frac{\left[z_{i j k}-a_{j} e^{-b / S_{i}}\left(\Lambda\left(t_{k}\right)-\Lambda\left(t_{k-1}\right)\right)\right]^{2}}{\Lambda\left(t_{k}\right)-\Lambda\left(t_{k-1}\right)},
\end{aligned}
$$

where $\mathbf{Z}$ denotes the observed data. Taking the first partial derivatives of $\ln L(\Theta \mid \mathbf{Z})$ with respect to $a_{1}, a_{2}, \ldots, a_{N}$ and $\sigma_{B}^{2}$ yields

$$
\begin{aligned}
& \frac{\partial}{\partial a_{j}} \ln L(\Theta \mid \mathbf{Z}) \\
& =\frac{1}{\sigma_{B}^{2}} \sum_{k=1}^{L}\left[z_{i j k}-a_{j} e^{-b / S_{i}}\left(\Lambda\left(t_{k}\right)-\Lambda\left(t_{k-1}\right)\right)\right] e^{-b / S_{i}}, \\
& \frac{\partial}{\partial \sigma_{B}^{2}} \ln L(\Theta \mid \mathbf{Z}) \\
& =-\frac{m N L}{2 \sigma_{B}^{2}}+\frac{1}{2\left(\sigma_{B}^{2}\right)^{2}} \\
& \quad \times \sum_{i=1}^{m} \sum_{j=n_{i-1}+1}^{n_{i}} \sum_{k=1}^{L} \frac{\left[z_{i j k}-a_{j} e^{-b / S_{i}}\left(\Lambda\left(t_{k}\right)-\Lambda\left(t_{k-1}\right)\right)\right]^{2}}{\Lambda\left(t_{k}\right)-\Lambda\left(t_{k-1}\right)} .
\end{aligned}
$$

Then, for specified values of $a_{1}, a_{2}, \ldots, a_{N}$ and $\sigma_{B}^{2}$ and setting these two derivatives to zero, the results of the MLE for $(b, \theta)$ can be written as

$$
\begin{gathered}
\widehat{a}_{j}(b, \theta)=\frac{\sum_{k=1}^{L} z_{i j k}}{\sum_{k=1}^{L} e^{-b / S_{i}}\left(\Lambda\left(t_{k}\right)-\Lambda\left(t_{k-1}\right)\right)}, \\
\widehat{\sigma}_{B}^{2}(b, \theta) \\
\times \frac{1}{m N L} \sum_{i=1}^{m} \sum_{j=n_{i-1}+1}^{n_{i}} \sum_{k=1}^{L}\left(\left[z_{i j k}-\frac{\sum_{k=1}^{L} z_{i j k}}{\sum_{k=1}^{L}\left(\Lambda\left(t_{k}\right)-\Lambda\left(t_{k-1}\right)\right)}\right.\right. \\
\left.\quad \times\left(\Lambda\left(t_{k}\right)-\Lambda\left(t_{k-1}\right)\right)\right]^{2} \\
\left.\quad \times\left(\left(\Lambda\left(t_{k}\right)-\Lambda\left(t_{k-1}\right)\right)\right)^{-1}\right) .
\end{gathered}
$$

It can be observed that $\widehat{\sigma}_{B}^{2}(b, \theta)$ bears no relation with $b$. Therefore, if we use $\widehat{\sigma}_{B}^{2}(b, \theta)$ to derive and maximize the profile log-likelihood function for $(b, \theta)$, we cannot derive the estimate of $b$. This problem can be solved as follows. Let $\alpha_{i j}=a_{j} e^{-b / S_{i}}$ represent the drift parameter of product $j$ under stress $i$; by substituting (22) into (20), the profile loglikelihood function for $\theta$ can be obtained as follows:

$$
\begin{aligned}
\ln L(\theta \mid \mathbf{Z})= & -\frac{m N L}{2}\left(\ln (2 \pi)+\ln \left(\widehat{\sigma}_{B}^{2}(b, \theta)\right)\right) \\
& -\frac{m N}{2} \sum_{k=1}^{L} \ln \left(\Lambda\left(t_{k}\right)-\Lambda\left(t_{k-1}\right)\right)-\frac{m N L}{2} .
\end{aligned}
$$

The MLE for $\theta$ can be obtained by maximizing the profile log-likelihood function in (23) through a onedimensional search. In this paper, the MATLAB function "FMINSEARCH" is used to find the estimates of $\theta$. Then, the estimates of $\alpha_{i j}$ and $\sigma_{B}^{2}$ can be obtained by substituting the estimates of $\theta$ into (22). Similarly, based on the distribution that $\alpha_{i j} \sim N\left(\mu_{a} e^{-b / S_{i}}, \sigma_{a}^{2} e^{-2 b / S_{i}}\right)$, using the MLE method for the estimated $\alpha_{i j}$ can obtain the estimates of $\left(\mu_{a}, \sigma_{a}^{2}, b\right)$. Thus, the final estimates of $\Phi$ can be derived.

4.2. SSADT. The MLE for the SSADT is similar to that of the CSADT. Assume that there are $N$ tested products for testing and the random Arrhenius model is utilized to model the relationship between the drift parameter and the stress level. Generally speaking, the degradation value is measured when the stress changes, and thus it is assumed that at time $\left(t_{i}\right)$ the measurement is implemented. Then, let $z_{i j k}$ denote 
the increment value for the $j$ th product at the $k$ th measurement time $\left(\tau_{k}\right)$ under stress $S_{i}$; we have

$$
\begin{gathered}
z_{i j k}=X_{S S}^{j}\left(\Lambda\left(\tau_{k}\right)\right)-X_{S S}^{j}\left(\Lambda\left(\tau_{k-1}\right)\right) \\
=\lambda_{i}\left(\Lambda\left(\tau_{k}\right)-\Lambda\left(\tau_{k-1}\right)\right)+\sigma_{B} B\left(\Lambda\left(\tau_{k}\right)-\Lambda\left(\tau_{k-1}\right)\right), \\
\zeta_{i}=\sum_{j=1}^{i} \varphi_{j} ; \\
t_{i-1}<\tau_{k} \leq t_{i} ; \quad t_{i}=\tau_{\zeta_{i}} ; \quad i=1,2, \ldots, m ; \\
j=1,2, \ldots, N ; \quad k=\zeta_{i-1}+1, \quad \zeta_{i-1}+2, \ldots, \zeta_{i},
\end{gathered}
$$

where $\varphi_{j}$ denotes the number of measurements under stress $S_{i}$ and $\zeta_{i}$ denotes the cumulative number of measurements up to stress $S_{i}$. It follows that $L=\zeta_{m}$. Based on the independent increment property of the Wiener process, the $z_{i j k}$ obeys the normal distribution as follows:

$$
\begin{array}{r}
z_{i j k} \sim N\left(a_{j} S_{i}^{b}\left(\Lambda\left(\tau_{k}\right)-\Lambda\left(\tau_{k-1}\right)\right), \sigma_{B}^{2}\left(\Lambda\left(\tau_{k}\right)-\Lambda\left(\tau_{k-1}\right)\right)\right) \\
i=1,2, \ldots, m ; \quad j=1,2, \ldots, N \\
k=\zeta_{i-1}+1, \quad \zeta_{i-1}+2, \ldots, \zeta_{i} .
\end{array}
$$

Then, the log-likelihood function can be expressed as

$$
\begin{aligned}
\ln L(\Theta \mid \mathbf{Z}) \\
=-\frac{m N L}{2}\left(\ln (2 \pi)+\ln \left(\sigma_{B}^{2}\right)\right) \\
\quad-\frac{N}{2} \sum_{i=1}^{m} \sum_{k=\zeta_{i-1}+1}^{\zeta_{i}} \ln \left(\Lambda\left(\tau_{k}\right)-\Lambda\left(\tau_{k-1}\right)\right) \\
\quad-\frac{1}{2 \sigma_{B}^{2}} \sum_{i=1}^{m} \sum_{j=1}^{N} \sum_{k=\zeta_{i-1}+1}^{\zeta_{i}} \frac{\left[z_{i j k}-a_{j} S_{i}^{b}\left(\Lambda\left(\tau_{k}\right)-\Lambda\left(\tau_{k-1}\right)\right)\right]^{2}}{\Lambda\left(\tau_{k}\right)-\Lambda\left(\tau_{k-1}\right)} .
\end{aligned}
$$

By taking the first partial derivatives of $\ln L(\Theta \mid \mathbf{Z})$ with respect to $a_{1}, a_{2}, \ldots, a_{N}$ and $\sigma_{B}^{2}$ and setting the two derivatives to zero, we have

$$
\begin{aligned}
\widehat{a}_{j}(b, \theta) & =\frac{\sum_{i=1}^{m} \sum_{k=\zeta_{i-1}+1}^{\zeta_{i}} z_{i j k} S_{i}^{b}}{\sum_{i=1}^{m} \sum_{k=\zeta_{i-1}+1}^{\zeta_{i}} S_{i}^{2 b}\left(\Lambda\left(\tau_{k}\right)-\Lambda\left(\tau_{k-1}\right)\right)}, \\
\widehat{\sigma}_{B}^{2}(b, \theta)= & \frac{1}{m N L} \\
& \times \sum_{i=1}^{m} \sum_{j=1}^{N} \sum_{k=\zeta_{i-1}+1}^{\zeta_{i}} \frac{\left[z_{i j k}-\widehat{a}_{j}(b, \theta) S_{i}^{b}\left(\Lambda\left(\tau_{k}\right)-\Lambda\left(\tau_{k-1}\right)\right)\right]^{2}}{\Lambda\left(\tau_{k}\right)-\Lambda\left(\tau_{k-1}\right)} .
\end{aligned}
$$

Substituting (27) into (26), the profile log-likelihood function for $(b, \theta)$ can be obtained as follows:

$$
\begin{aligned}
\ln L(b, \theta \mid \mathbf{Z})= & -\frac{m N L}{2}\left(\ln (2 \pi)+\ln \left(\widehat{\sigma}_{B}^{2}(b, \theta)\right)\right) \\
& -\frac{N}{2} \sum_{i=1}^{m} \sum_{k=\zeta_{i-1}+1}^{\zeta_{i}} \ln \left(\Lambda\left(\tau_{k}\right)-\Lambda\left(\tau_{k-1}\right)\right) \\
& -\frac{m N L}{2}
\end{aligned}
$$

Similar to the MLE of the CSADT, maximizing the profile $\log$-likelihood function in (28) gives the MLE for $(b, \theta)$. Then, the final estimates of $a_{1}, a_{2}, \ldots, a_{N}$ and $\sigma_{B}^{2}$ can be obtained. Finally, the $\left(\mu_{a}, \sigma_{a}^{2}\right)$ can be derived by fitting the estimated $a_{1}, a_{2}, \ldots, a_{N}$.

\section{Experimental Studies}

In this section, we provide a simulation study and a case study to demonstrate the application of the proposed ADT model with random effects and compare the performance of our ADT model with the ADT model without considering the random effects. To compare the fitting of the proposed models, the Akaike information criterion (AIC) is used. AIC deals with the trade-off between the goodness of model fitting and the complexity of the model, which can be formulated as

$$
\mathrm{AIC}=2 p-2 \ln L
$$

where $p$ is the number of parameters and $L$ is the maximum likelihood.

5.1. A Simulation Study. In this experiment, we use the nonlinear Wiener process to simulate the degradation in terms of the fatigue crack growth in metals for the SSADT. The simulation is based on the fatigue crack growth data described by Lu and Meeker [33], which is originally presented by Hudak et al. [34]. The fatigue crack growth data contains 21 products which are tested until 0.12 million cycles with measurement interval of 0.01 million cycles or until the crack size reaches the critical length of 1.6 inches and results in failure. A similar simulation study based on this fatigue crack growth data for accelerated tests modelling has also been performed in [35].

To illustrate the application of the proposed model, data are simulated based on the random power rule model for three stress levels. The normal level of $S_{0}=1$ represents the condition of the example data presented in [33]. The three levels used for simulation are $S_{1}=1.2, S_{2}=1.4$, and $S_{3}=1.6$. By using the MLE method in [36] and setting that $b=1$ in the random power rule model, we can obtain the estimated values of the fatigue crack growth data. The estimated values given in the second line of Table 1 are regarded as the real values to simulate the degradation data. 15 products are simulated with measurement frequency 
of 0.003 million cycles and terminated time of 0.09 million cycles, and there are 0.03 million cycles in each stress. The initial value of the degradation data is assumed to be 0 ; thus, the failure threshold is 0.7. The simulated data are shown in Figure 2. Compared with the practical cases, an advantage of the simulations is that the real values of the model parameters exist.

For simplicity, the model proposed in this paper is referred to as $M_{1}$ and the model presented by [10,11] as $M_{2}$. To compare these two models, Table 1 shows the estimation results of the parameters, the log-likelihood function value, the AIC, and the $95 \%$ confidence interval (CI). From Table 1, it can be observed that the estimated parameters by $M_{1}$ are much closer to the real results than that by $M_{2}$. Moreover, $M_{1}$ obtains higher Log-LF and less AIC compared with $M_{2}$. This implies that the proposed model has better model fit than $M_{2}$.

To further compare these models, we give the PDFs and CDFs in Figure 3. It is shown that the PDF and CDF of $M_{1}$ are more close to the real PDF and CDF. The results demonstrate that if the random effects are not considered in the ADT modeling, it could result in sharper PDF and narrower confident interval. Therefore, some failure probability is ignored. If the failure probability in the interval [ $\left[\begin{array}{ll}0.8 & 0.9\end{array}\right]$ is ignored, it could lead to hysteretic maintenance and then increase the failure risk at the early time. Additionally, if the failure probability in the interval [1.31.8] is ignored, it could result in premature maintenance and then reduce the utilization rate of the products. The reason why $M_{1}$ has a better performance than $M_{2}$ can be explained as follows. If the random effects are not considered in the degradation modeling, the random effects are represented by the uncertainty of the Wiener process, which is different from that of the uncertainty of the random effects. This leads to weak modeling fit and poor accuracy of lifetime estimation. Therefore, it is necessary to consider the random effects in the degradation modeling.

5.2. A Case Study. As a solid-state lighting source, the light emitting diodes (LEDs) have been increasingly used in display backlighting, communications, medical services, signage, and general illumination [37-39]. The light intensity of a LED decreases over time, leading to a soft failure when its light intensity drops below a critical threshold level. We use the dataset of the CSADT presented in [40] for an illustrative purpose. To derive the lifetime distribution on the design level under $25 \mathrm{~mA}, 24$ units are put into test under constant accelerated levels of electric current, which are $I_{1}=35 \mathrm{~mA}$ and $I_{1}=40 \mathrm{~mA}$. The degradation level of each unit was measured every 50 hours until 250 hours. The degradation data are depicted in Figure 4. More details of the degradation data can be found in [40]. Following the engineering routine, the failure threshold level is set as 50 .

According to $[12,40]$, the degradation rate of LEDs regarding the electric current can be described by the power law model. Thus, we use the random power law model to represent the acceleration model. Based on the MLE method presented in Section 4.1, we can obtain the estimation results of the parameters, the log-likelihood function value, the AIC, and the $95 \%$ CI as shown in Table 2. For the estimated $\theta$,

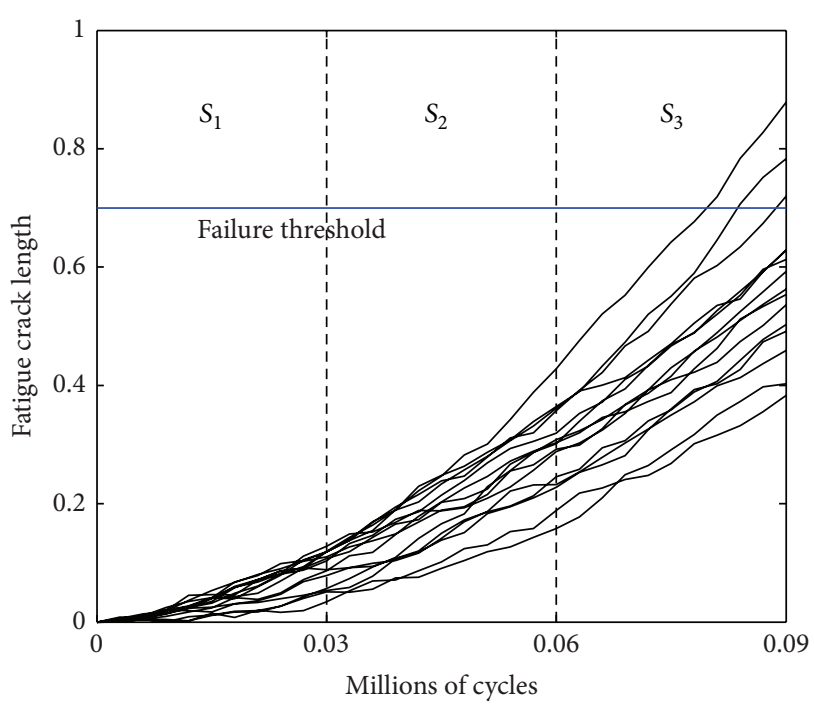

Figure 2: The simulated data.

the nonlinear characteristic is clearly confirmed. In comparison, Table 2 shows that $M_{1}$ clearly outperforms $M_{2}$ in terms of the Log-LF and AIC. It indicates that the proposed model has better model fit than $M_{2}$.

To further test the validity of the ADT model with random effects, we give the normal probability plot for the LED data, as shown in Figure 5. It can be observed that $M_{1}$ shows a better modeling fit than $M_{2}$. Additionally, we verify the normality assumption of Wiener process for the LED data by the JB test method [41]. This implies the appropriateness of using the Wiener process.

In the following, we plot the estimated mean degradation paths based on the two models in conjunction with the sample average in Figure 6. The estimating curves by the two models tally quite well. We further depict the PDFs and CDFs based on the two models in Figure 7 by transforming the degradation under the design level under $25 \mathrm{~mA}$. It can be observed that the PDF by $M_{1}$ is sharper than that of $M_{2}$. It indicates that whether considering the random effects has little impact on the estimation of the mean time to failure (MTTF) but has distinct impact on the distribution of failure time.

Overall, the simulation study and the practical study demonstrate that the proposed model can work well and efficiently. If the random effects are not considered, some failure probability could be ignored, which leads to the failure maintenance or the premature maintenance. Therefore, it is necessary to consider the random effects.

\section{Conclusions}

In this paper, we study the problem of modeling the accelerated degradation processes based on the nonlinear Wiener process with random effects. In order to represent the product-to-product variability among different individuals of the accelerated degradation processes, we regard the drift parameter of the nonlinear Wiener process as the random 
TABLE 1: Comparisons of two degradation models with the simulated degradation data.

\begin{tabular}{lcccccccc}
\hline & $\mu_{a}$ & $\sigma_{a}^{2}$ & $b$ & $\sigma_{B}^{2}$ & $\theta$ & Log-LF & AIC & 95\% CI \\
\hline Real value & 17.3 & 28.6 & 0.7 & $8.57 e-2$ & 1.50 & - & - & {$[0.0852,0.223]$} \\
$M_{1}$ & 19.0 & 18.2 & 0.70 & $8.53 e-2$ & 1.56 & 1548 & -3087 & {$[0.0930,0.177]$} \\
$M_{2}$ & 20.1 & - & 1.01 & 0.117 & 1.58 & 1492 & -2976 & {$[0.106,0.133]$} \\
\hline
\end{tabular}

TABLE 2: Comparisons of two degradation models with the degradation data of LEDs.

\begin{tabular}{ccccccccc}
\hline & $\mu_{a}$ & $\sigma_{a}^{2}$ & $b$ & $\sigma_{B}^{2}$ & $\theta$ & Log-LF & AIC & 95\% CI \\
\hline$M_{1}$ & $3.25 e-05$ & $2.06 e-11$ & 3.14 & 5.84 & 0.450 & -317 & -644 & {$[1672,53466]$} \\
$M_{2}$ & $1.83 e-05$ & - & 3.30 & 6.24 & 0.448 & -320 & -648 & {$[1848,57202]$} \\
\hline
\end{tabular}

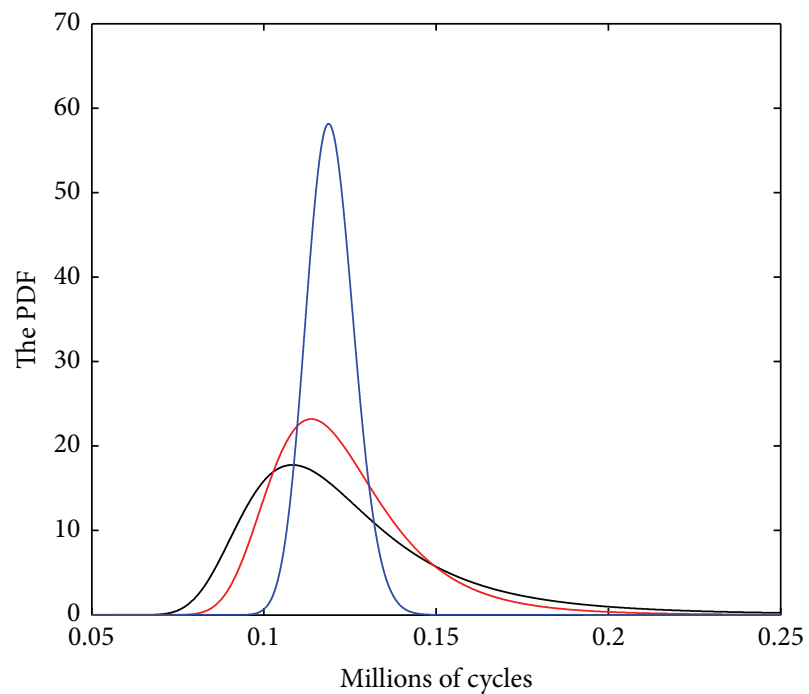

- Real value
$M_{1}$

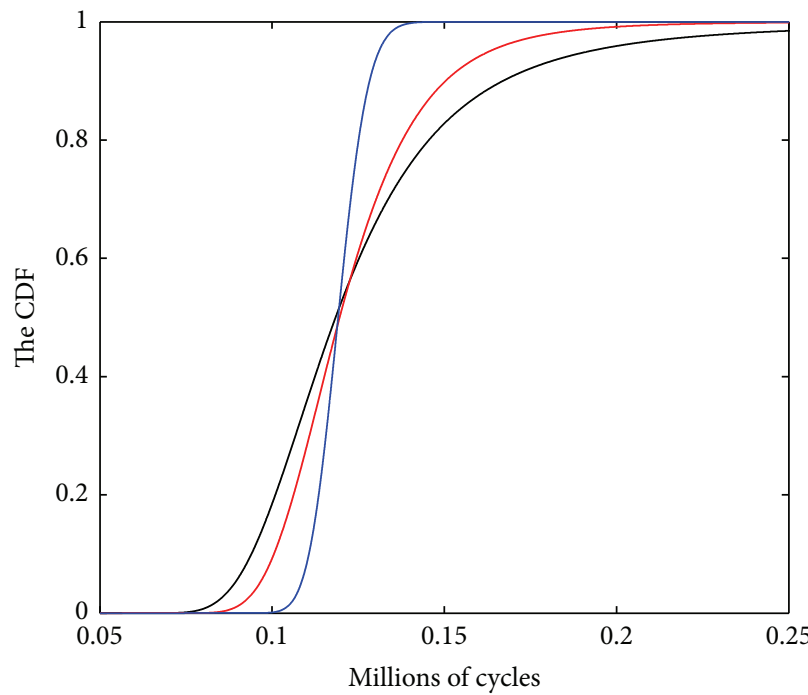

- Real value

- $M_{1}$

(a)

(b)

FIgURE 3: The estimated PDFs and CDFs by $M_{1}$ and $M_{2}$.

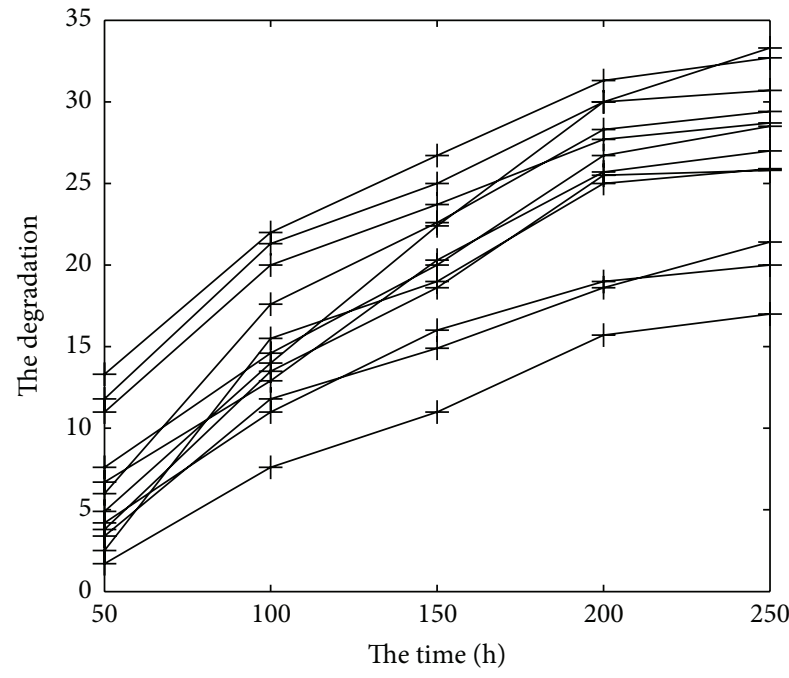

(a)

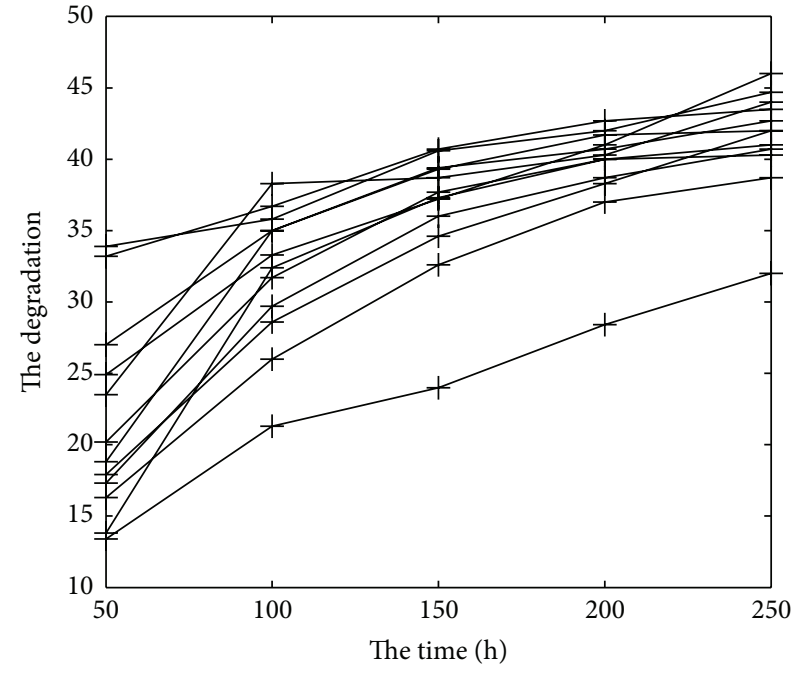

(b)

FIgURE 4: Degradation paths of LED. ((a) $I_{1}=35 \mathrm{~mA}$; (b) $I_{1}=40 \mathrm{~mA}$.) 


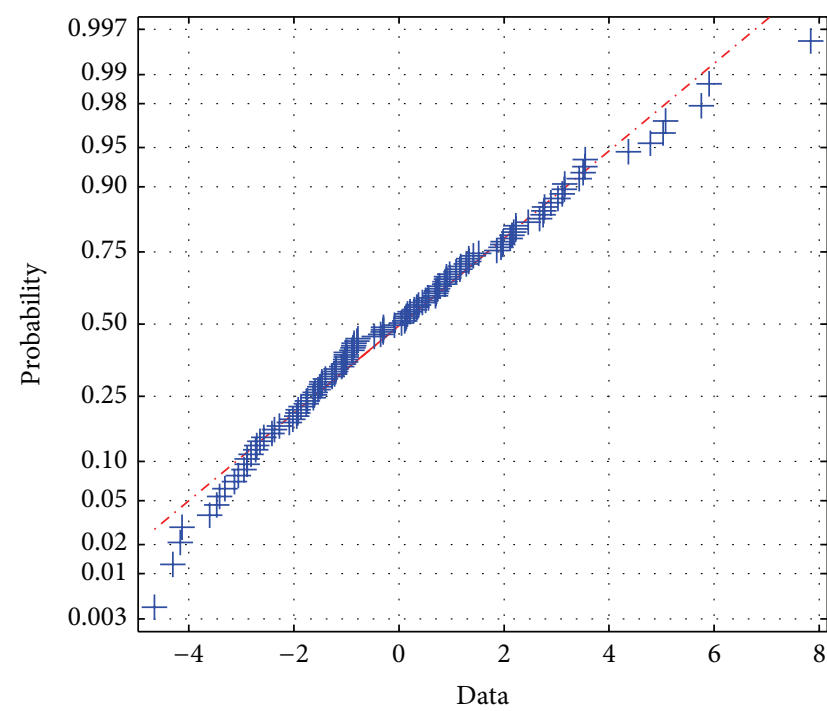

(a)

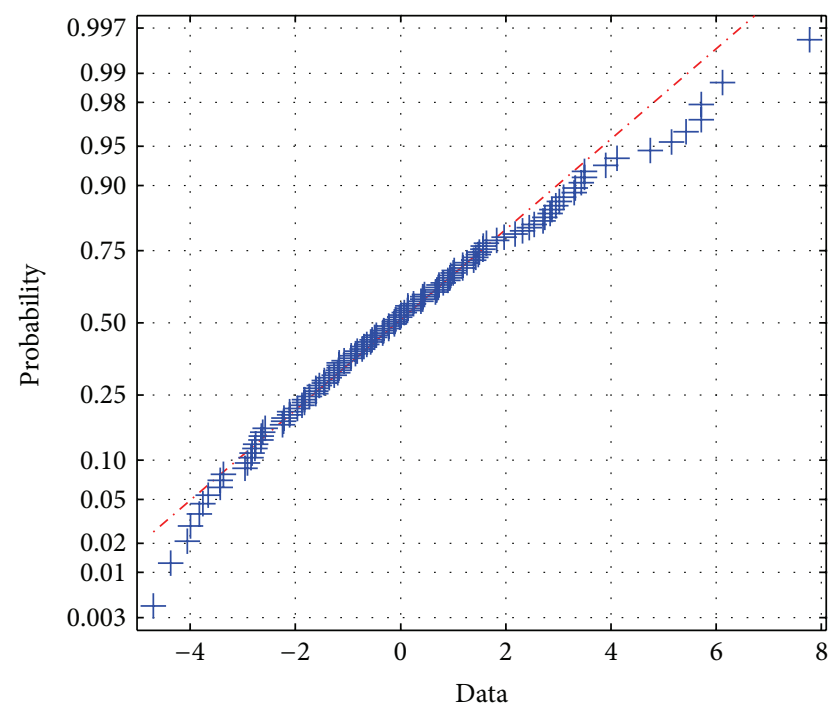

(b)

Figure 5: The normal probability plot for the LED data. ((a) $M_{1}$; (b) $M_{2}$.)

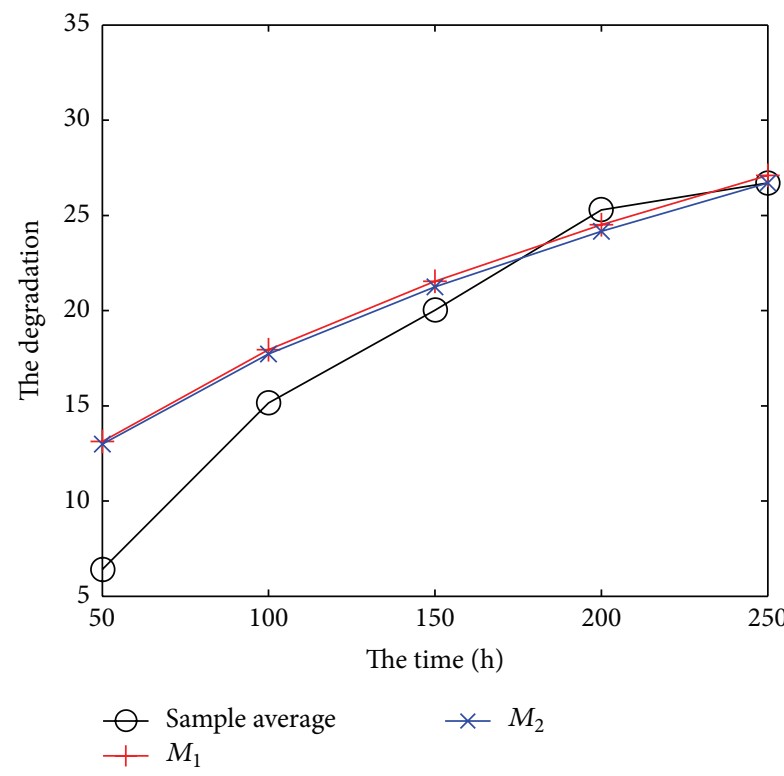

(a)

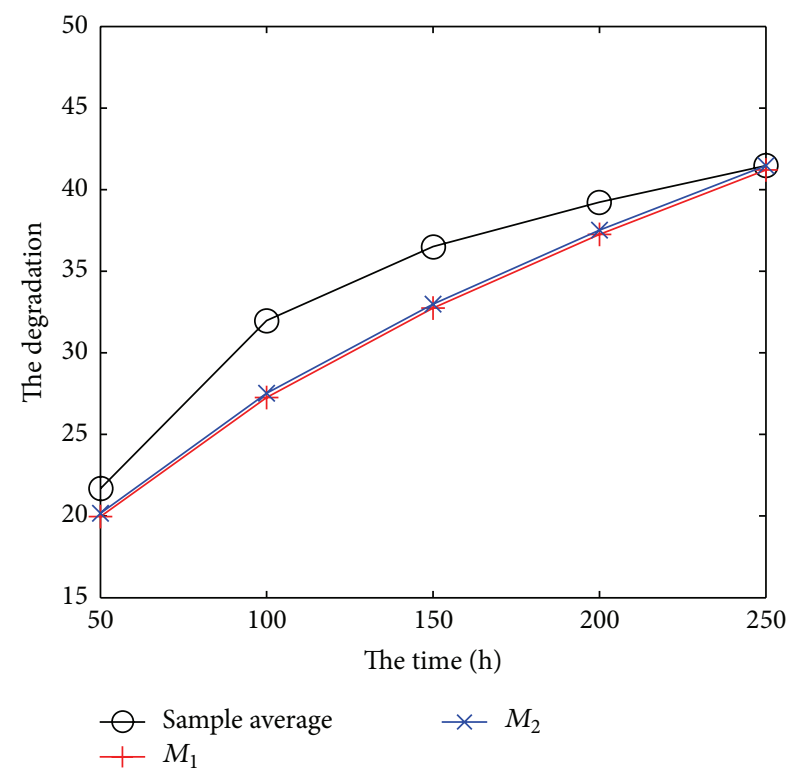

(b)

Figure 6: Estimated mean paths. ((a) $I_{1}=35 \mathrm{~mA}$; (b) $I_{1}=40 \mathrm{~mA}$.)

parameter. Firstly, we derive the lifetime distribution of the nonlinear Wiener process with random effects. Secondly, the random acceleration model is generalized to the nonlinear case, and the random power rule model and random Eyring model are presented. Then, the random effects are incorporated into the CSADT and SSADT. The unknown parameters in the degradation models are obtained by using the twostep MLE method. A simulation study and a case study with comparison to the existing model under the same conditions show the superiorities of the proposed model. The results demonstrate that it is necessary to incorporate the random effects into the accelerated degradation modeling.

We primarily discuss the issues associated with the accelerated degradation modeling in this paper. Some other issues need to be further exploited. Firstly, we only use the time-transformed Wiener process to model ADT. However, the time-transformed Wiener process could not be appropriate for all the nonlinear degradation process. Therefore, using other types of nonlinear degradation process to model ADT data is needed. Secondly, the modeling of ADT data 


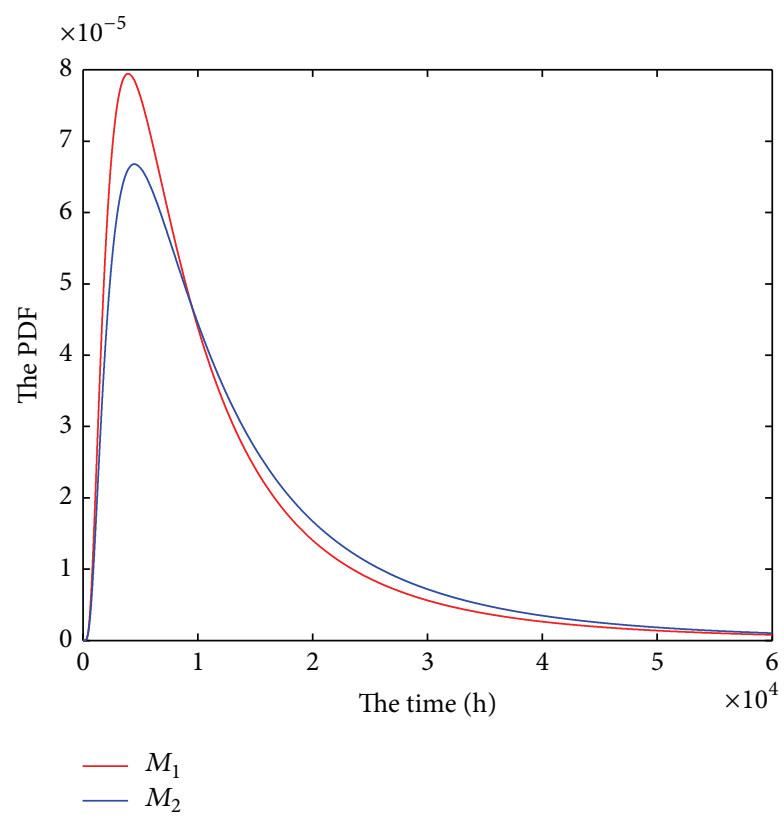

(a)

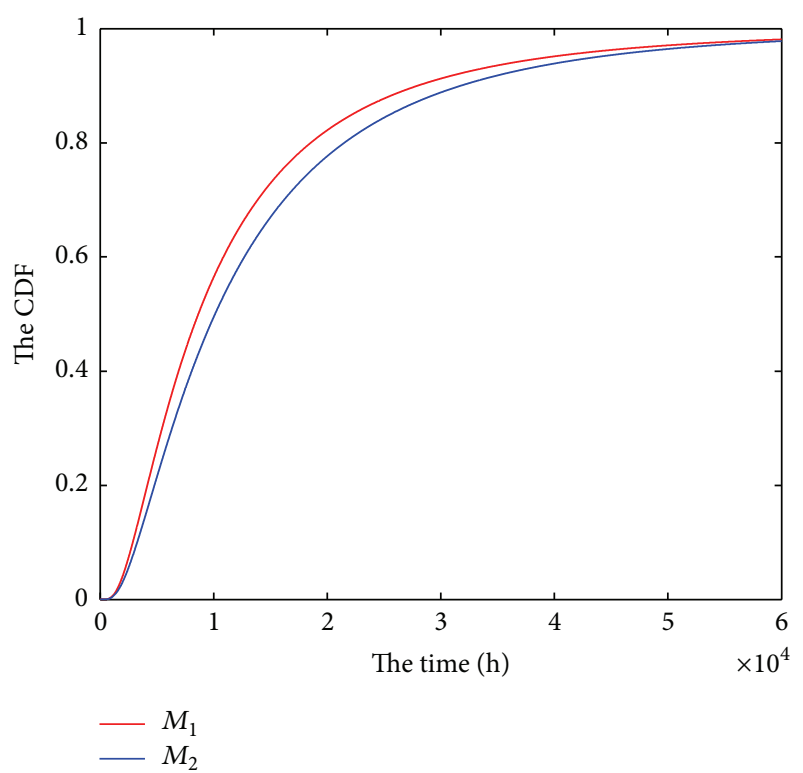

(b)

FIGURE 7: The estimated PDFs by $M_{1}$ and $M_{2}$.

presented in this paper is only under a single stress, and thus it is necessary to model the ADT data with multiple stresses. Thirdly, we only discuss the issues regarding how to model the ADT data. However, many researchers are concerned with how to conduct a degradation test efficiently. Thus, optimizing the degradation tests based on Wiener processes with random effects needs to be further researched. Additionally, application in other practical cases is necessary in future research.

\section{Conflict of Interests}

The authors declare that there is no conflict of interests regarding the publication of this paper.

\section{Acknowledgments}

The authors thank the editor and three anonymous reviewers for their valuable and constructive suggestions that led to considerable improvements of this paper. This work was supported by the National Science Foundation of China under Grants nos. 41174162, 61374138, 61004069, and 61370031.

\section{References}

[1] W. Q. Meeker, L. A. Escobar, and C. J. Lu, "Accelerated degradation tests: modeling and analysis," Technometrics, vol. 40, no. 2, pp. 89-99, 1998.

[2] N. Z. Gebraeel, M. A. Lawley, R. Li, and J. K. Ryan, "Residual-life distributions from component degradation signals: a Bayesian approach," IIE Transactions, vol. 37, no. 6, pp. 543-557, 2005.
[3] Z.-S. Ye, Y. Shen, and M. Xie, "Degradation-based burn-in with preventive maintenance," European Journal of Operational Research, vol. 221, no. 2, pp. 360-367, 2012.

[4] S.-T. Tseng, J. Tang, and I.-H. Ku, "Determination of burn-in parameters and residual life for highly reliable products," Naval Research Logistics (NRL), vol. 50, no. 1, pp. 1-14, 2003.

[5] C. Park and W. J. Padgett, "Stochastic degradation models with several accelerating variables," IEEE Transactions on Reliability, vol. 55, no. 2, pp. 379-390, 2006.

[6] X.-S. Si, W. Wang, C.-H. Hu, and D.-H. Zhou, "Remaining useful life estimation-a review on the statistical data driven approaches," European Journal of Operational Research, vol. 213, no. 1, pp. 1-14, 2011.

[7] C. Park and W. J. Padgett, "Accelerated degradation models for failure based on geometric Brownian motion and gamma processes," Lifetime Data Analysis, vol. 11, no. 4, pp. 511-527, 2005.

[8] H. Lim and B.-J. Yum, "Optimal design of accelerated degradation tests based on Wiener process models," Journal of Applied Statistics, vol. 38, no. 2, pp. 309-325, 2011.

[9] X. Wang, B. Guo, and Z. Cheng, "Residual life estimation based on bivariate Wiener degradation process with timescale transformations," Journal of Statistical Computation and Simulation, vol. 84, no. 3, pp. 545-563, 2014.

[10] C.-M. Liao and S.-T. Tseng, "Optimal design for step-stress accelerated degradation tests," IEEE Transactions on Reliability, vol. 55, no. 1, pp. 59-66, 2006.

[11] S. T. Tseng and C. Y. Peng, "Stochastic diffusion modeling of degradation data," Journal of Data Science, vol. 5, pp. 315-333, 2007.

[12] H. Liao and E. A. Elsayed, "Reliability inference for field conditions from accelerated degradation testing," Naval Research Logistics (NRL), vol. 53, no. 6, pp. 576-587, 2006. 
[13] L. Wang, R. Pan, X. Li, and T. Jiang, "A Bayesian reliability evaluation method with integrated accelerated degradation testing and field information," Reliability Engineering \& System Safety, vol. 112, pp. 38-47, 2013.

[14] C.-Y. Peng and S.-T. Tseng, "Mis-specification analysis of linear degradation models," IEEE Transactions on Reliability, vol. 58, no. 3, pp. 444-455, 2009.

[15] X. Wang, "Wiener processes with random effects for degradation data," Journal of Multivariate Analysis, vol. 101, no. 2, pp. 340-351, 2010.

[16] X. Si, W. Wang, C.-H. Hu, D.-H. Zhou, and M. G. Pecht, "Remaining useful life estimation based on a nonlinear diffusion degradation process," IEEE Transactions on Reliability, vol. 61, pp. 50-67, 2012.

[17] X. Wang, P. Jiang, B. Guo, and Z. Cheng, "Real-time reliability evaluation with a general Wiener process-based degradation model," Quality and Reliability Engineering International, vol. 30, no. 2, pp. 205-220, 2014.

[18] Z. Ye, Y. Wang, K. L. Tsui, and M. Pecht, "Degradation data analysis using Wiener processes with measurement errors," IEEE Transactions on Reliability, vol. 62, pp. 772-780, 2013.

[19] S. Tang, C. Yu, X. Wang, X. Guo, and X. Si, "Remaining useful life prediction of lithium-ion batteries based on the wiener process with measurement error," Energies, vol. 7, pp. 520-547, 2014.

[20] C.-C. Tsai, S.-T. Tseng, and N. Balakrishnan, "Optimal design for degradation tests based on gamma processes with random effects," IEEE Transactions on Reliability, vol. 61, pp. 604-613, 2012.

[21] S. Tang, X. Guo, Z. Zhou, X. Si, and Z. Zhou, "Step stress accelerated degradation process modeling and remaining useful life estimation," Journal of Mechanical Engineering. Under review (Chinese).

[22] G. Jin, D. E. Matthews, and Z. Zhou, "A Bayesian framework for on-line degradation assessment and residual life prediction of secondary batteries inspacecraft," Reliability Engineering \& System Safety, vol. 113, pp. 7-20, 2013.

[23] S. Tang, X. Guo, Z. Zhou, Z. Zhou, and B. Zhang, "Real time remaining useful life prediction based on nonlinear Wiener based degradation processes with measurement errors," Journal of Central South University. In press.

[24] X. Wang, N. Balakrishnan, and B. Guo, "Residual life estimation based on a generalized Wiener degradation process," Reliability Engineering \& System Safety, vol. 124, pp. 13-23, 2014.

[25] K. A. Kaiser and N. Z. Gebraeel, "Predictive maintenance management using sensor-based degradation models," IEEE Transactions on Systems, Man, and Cybernetics A: Systems and Humans, vol. 39, no. 4, pp. 840-849, 2009.

[26] Z. Ye, N. Chen, and K. L. Tsui, "A Bayesian approach to condition monitoring with imperfect inspections," Quality and Reliability Engineering International, 2013.

[27] G. A. Whitmore and F. Schenkelberg, "Modelling accelerated degradation data using Wiener diffusion with a time scale transformation," Lifetime Data Analysis, vol. 3, pp. 27-45, 1997.

[28] J. L. Folks and R. S. Chhikara, "The inverse Gaussian distribution and its statistical application-a review," Journal of the Royal Statistical Society B. Methodological, vol. 40, no. 3, pp. 263-289, 1978.

[29] A. Høyland and M. Rausand, System Reliability Theory: Models and Statistical Methods, vol. 420, John Wiley \& Sons, New York, NY, USA, 1994.
[30] J. Zhang, T. Zhou, H. Wu, L. Yu, W. Wu, and J. Ren, "Constantstep-stress accelerated life test of white OLED under weibull distribution case," IEEE Transactions on Electron Devices, vol. 59, pp. 715-720, 2012.

[31] W. Luo, C. H. Zhang, X. Chen, and Y. Y. Tan, "Reliability demonstration based on accelerated degradation testing for unknown model parameters," Proceedings of the Institution of Mechanical Engineers Part O-Journal of Risk and Reliability, vol. 227, pp. 162-172, 2013.

[32] W. Chen, J. Liu, L. Gao, J. Pan, and X. Lu, "Step-stress accelerated degradation test modeling and statistical analysis methods," Chinese Journal of Mechanical Engineering, vol. 26, pp. 11541159, 2013.

[33] C. J. Lu and W. Q. Meeker, "Using degradation measures to estimate a time-to-failure distribution," Technometrics, vol. 35, no. 2, pp. 161-174, 1993.

[34] S. Hudak Jr., A. Saxena, R. Bucci, and R. Malcolm, "Development of standard methods of testing and analyzing fatigue crack growth rate data," DTIC Document AFML-TR-78-40, Westinghouse R \& D Center, Westinghouse Electric Corporation, Pittsburgh, Pa, USA, 1978.

[35] W. J. Padgett and M. A. Tomlinson, "Inference from accelerated degradation and failure data based on Gaussian process models," Lifetime Data Analysis, vol. 10, no. 2, pp. 191-206, 2004.

[36] G. A. Whitmore, "Estimating degradation by a wiener diffusion process subject to measurement error," Lifetime Data Analysis, vol. 1, no. 3, pp. 307-319, 1995.

[37] M.-H. Chang, D. Das, P. V. Varde, and M. Pecht, "Light emitting diodes reliability review," Microelectronics Reliability, vol. 52, no. 5, pp. 762-782, 2012.

[38] P. Liu, R. Wu, Z. Zheng, H. Li, and X. Liu, "Optimized design of LED freeform lens for uniform circular illumination," Journal of Zhejiang University SCIENCE C, vol. 13, pp. 929-936, 2012.

[39] R. Wu, P. Liu, Y. Zhang, Z. Zheng, H. Li, and X. Liu, "Ray targeting for optimizing smooth freeform surfaces for LED non-rotational illumination," Journal of Zhejiang University SCIENCE C, vol. 14, pp. 785-791, 2013.

[40] V. N. H. Chaluvadi, Accelerated life testing of electronic revenue meters [M.S. thesis], Clemson University, Clemson, SC, USA, 2008.

[41] A. K. Bera and C. M. Jarque, "Efficient tests for normality, homoscedasticity and serial independence of regression residuals: Monte Carlo Evidence," Economics Letters, vol. 7, pp. 313318, 1981. 


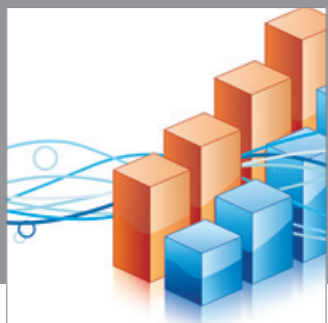

Advances in

Operations Research

mansans

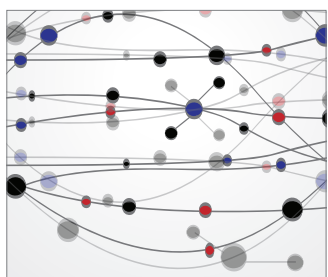

The Scientific World Journal
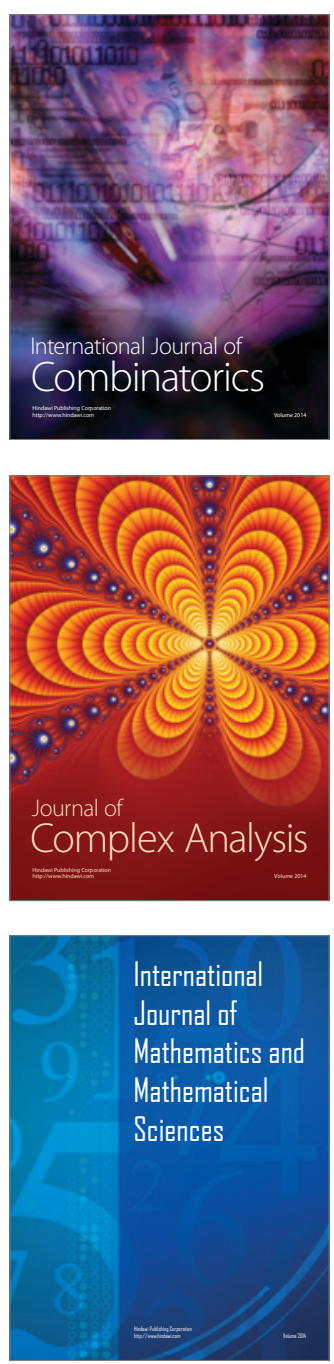
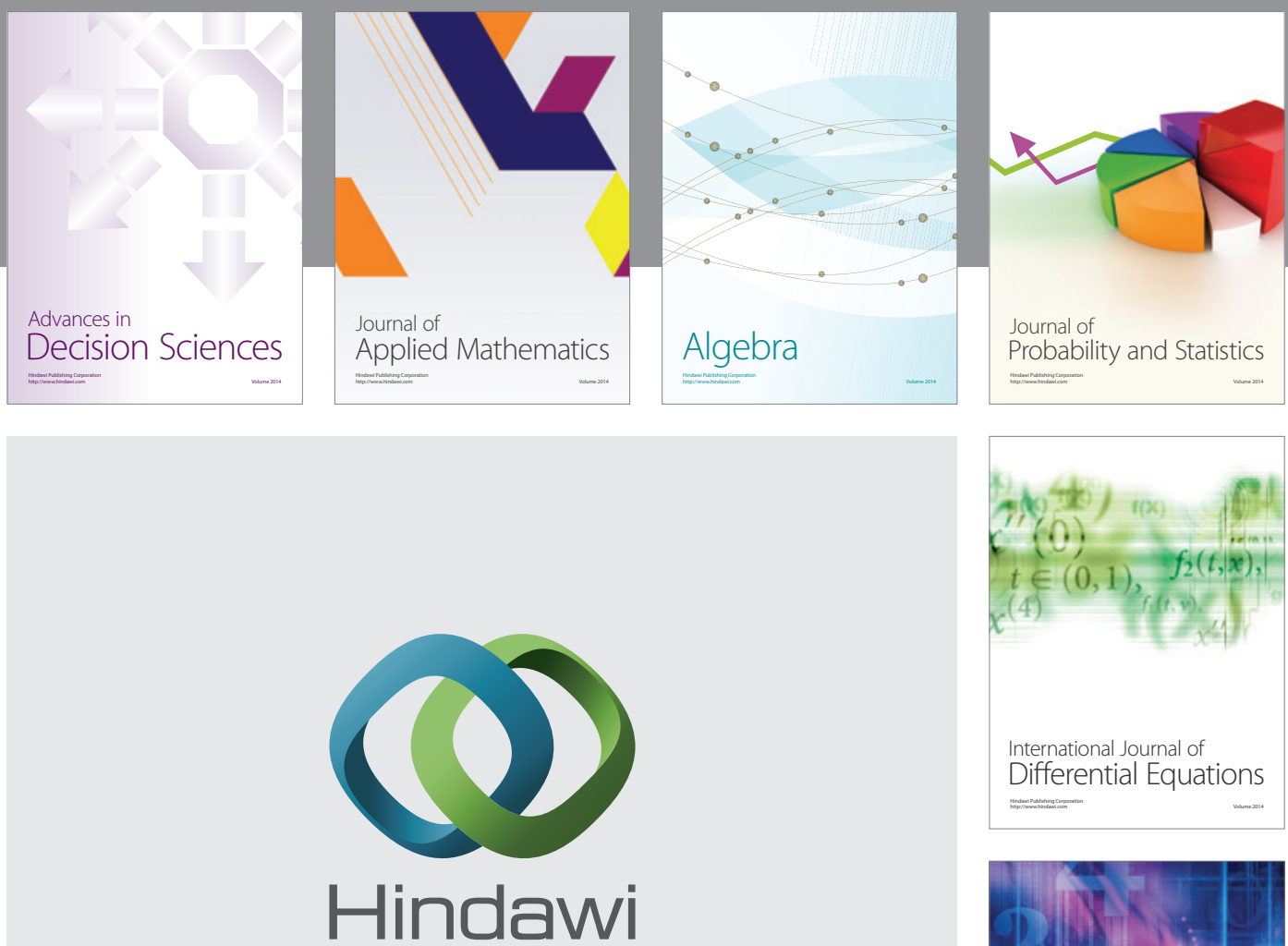

Submit your manuscripts at http://www.hindawi.com
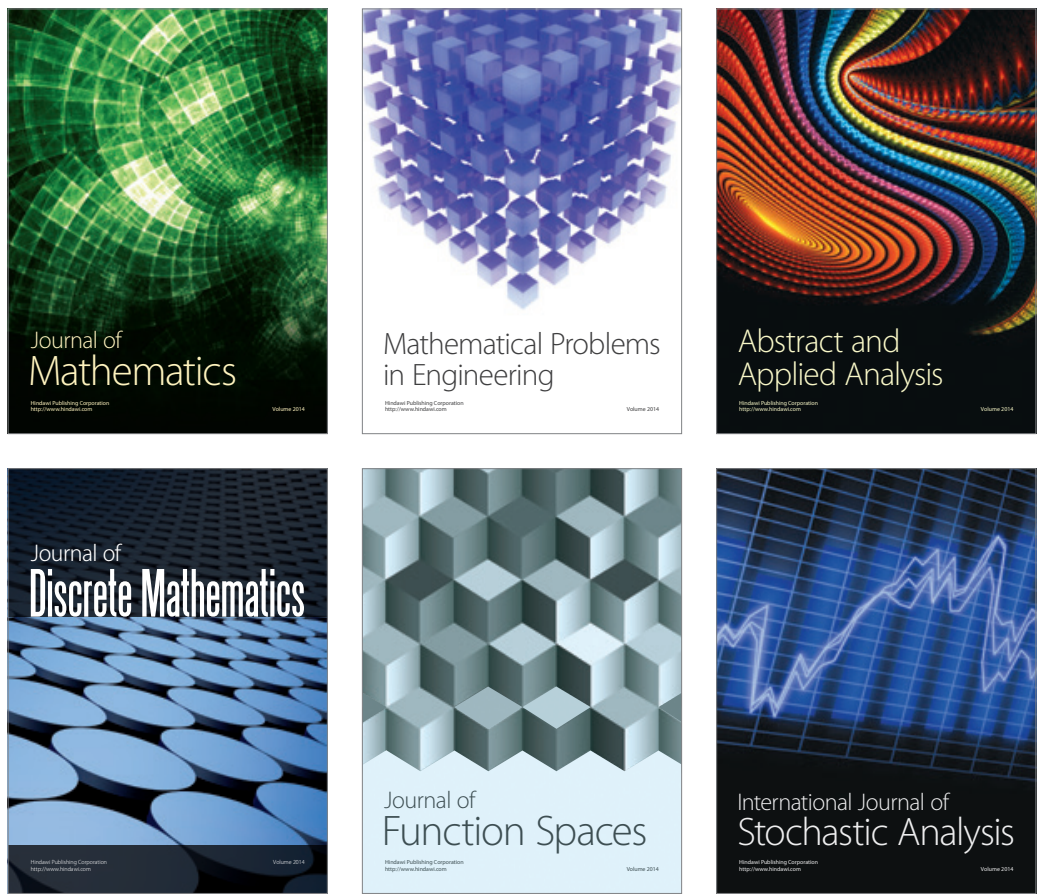

Journal of

Function Spaces

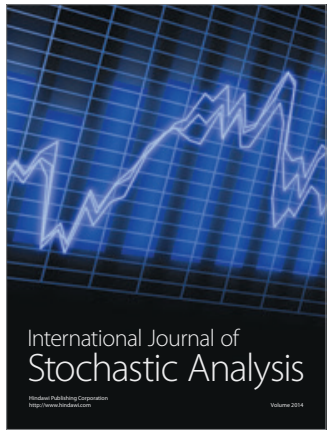

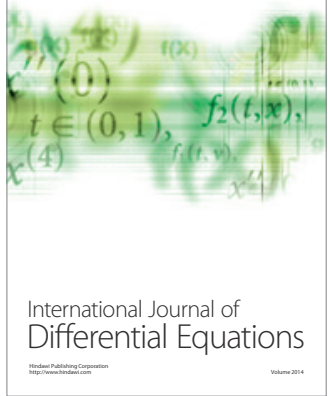
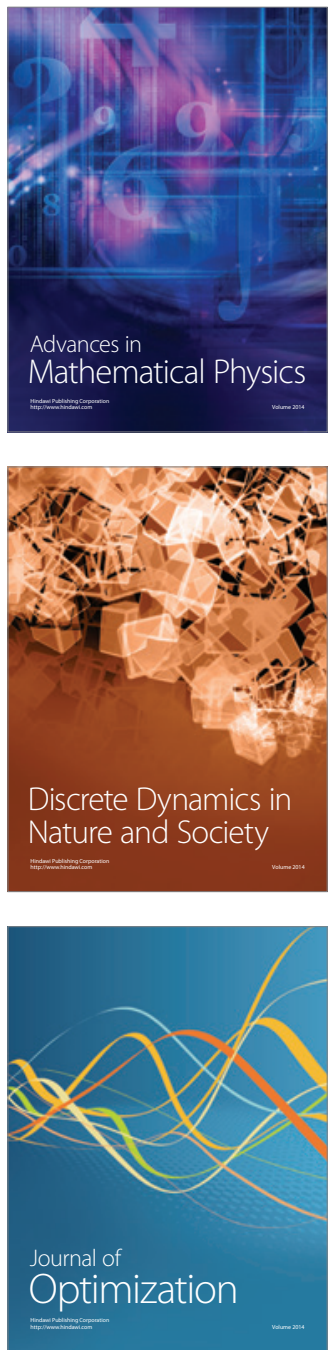